\title{
Effect of Pravastatin on the Incidence of Preeclampsia
}

\author{
Mohamed S. Hassanain, Bassem R. Abdel-Aziz, Mohamed A. Elsayed \\ Department of Obstetrics and Gynecology, Faculty of Medicine, Al-Azhar University
}

Corresponding author: Mohamed A. Elsayed; Mobile: 01140407230; Email: m_atef_dr@yahoo.com

\begin{abstract}
Background: Preeclampsia is a disorder of widespread vascular endothelial malfunction and vasospasm that occurs after 20 weeks' gestation and can present as late as 4-6 weeks post-partum. It is clinically defined by hypertension and proteinuria, with or without pathologic edema. Aim of the Work: is to study the effect of pravastatin given for primigravidae from $13^{\text {th }}$ week to $16^{\text {th }}$ week of gestation on the incidence of preeclampsia compared to a control group in Egyptian women. Subjects and methods: This prospective case control study included 400 pregnant women presenting for routine antenatal care at $13^{\text {th }}$ to $16^{\text {th }}$ weeks' gestation at EL Sayed Galal Obstetric outpatient clinic, Al-Azhar University. Results: In our study, 16 cases developed preeclampsia in the control group (11 cases were severe and the remaining 5 were mild) with a percentage of $8 \%$ instead of 6 cases in the study group ( 4 cases were severe and 2 were mild) with a percentage of $3 \%$.

Conclusion: Pravastatin can be used as an effective agent in the prevention of preeclampsia in newborns within neonatal period including fetal weight, gestational age at time of delivery and NICU admission. However, extended follow up is needed till the age of 5 years to assess cognitive functions and school performance among children born for women who received pravastatin for prevention of preeclampsia.
\end{abstract}

Keywords: pravastatin, preeclampsia, primigravida, gestation

\section{Introduction}

Preeclampsia is a pregnancy-specific disorder that affects 2 to $8 \%$ of all pregnancies and remains a leading cause of maternal and perinatal morbidity and mortality worldwide. Diagnosis is based on new onset of hypertension and proteinuria. Multiple organ systems can be affected with severe disease. The wide range of risk factors reflects the heterogeneity of preeclampsia. Obesity, which is increasing at an alarming rate, is also a risk factor for preeclampsia as well as for later life cardiovascular disease. Exploring common features may provide insight into the pathophysiologic mechanisms underlying preeclampsia ${ }^{(\mathbf{1})}$.

Hypertension developing in the second half of pregnancy is subdivided according to the presence or absence of co-existing significant proteinuria into preeclampsia and gestational hypertension. An evidence suggests that preeclampsia can be further subdivided into early preeclampsia and late preeclampsia with the former being associated with a higher incidence of fetal growth restriction and both short-term and long-term maternal mortality and morbidity ${ }^{(2)}$.

There is no proven effective method for the prevention of preeclampsia (PE), nevertheless, routine prenatal care in the last 50 years has evolved with the aim of early identification of women at high-risk for preeclampsia, which could potentially improve pregnancy outcome. Intensive maternal and fetal monitoring in such patients would lead to an earlier diagnosis of the clinical signs of the disease and the associated fetal growth restriction and avoid the development of serious complications through such interventions as the administration of antihypertensive medication and early delivery. Early identification of the high-risk group for the development of PE is also important for future studies investigating the potential role of pharmacological interventions starting from the first trimester to improve placentation and reduce the prevalence of the disease ${ }^{(3)}$.

Pravastatin is one of the leading and most effective statins derived from the natural product compactin. Statins are successful widely used drugs that decrease the risk of coronary heart diseases and strokes by lowering cholesterol levels. They selectively inhibit the key regulatory enzyme of the cholesterol synthesis pathway, thus lowering level of plasma LDL (bad) cholesterol ${ }^{(4)}$.

Pravastatin is a competitive inhibitor of 3 hydroxy-3-methyl-glutaryl-coenzyme A reductase, the enzyme that catalyzes the rate-limiting step in cholesterol synthesis. It is the most hydrophilic polar compound among the current statins, and thus thought to have limited ability to cross the placenta. In addition it is the least potent sterol synthesis inhibitor ${ }^{(5)}$.

The Medical Genetics branch of the National Institutes of Health reviewed 214 
Mohamed Hassanain et al.

ascertained pregnancy exposures to statins that were reported to the U.S. Food and Drug Administration (FDA) from 1987 to 2001. Of the 70 evaluable cases reviewed in the final report (20) cases of pravastatin exposure were included ${ }^{(6)}$.

Exploration of two large case control studies of birth defects, the National Birth Defects Prevention Study and the Slone Epidemiology Center Birth Defects Study failed to show any link between statin exposure and a pattern of birth defects ${ }^{(7)}$.

In addition, a prospective observational cohort study by the Motherisk program in Toronto did not find any malformation patterns or increased malformations in infants of 64 women with first trimester exposure to statins compared with women without exposure to known teratogens ${ }^{(\mathbf{8})}$.

The properties and mechanisms of action of pravastatin make it a highly promising candidate for the prevention of preeclampsia. Using various animal models of preeclampsia, Saad and others have shown that pravastatin administration to rodents destined to develop preeclampsia improved their abnormal vascular profile, lowered blood pressure, restored angiogenic balance by reducing circulating sFlt-1 and soluble endoglin levels, and up-regulating vascular and placental growth factors. In addition, pravastatin up regulated endothelial nitric oxide synthase expression in the vasculature, restored heme oxygenase-1/carbon monoxide balance, and prevented kidney injury. These benefits were observed without affecting maternal cholesterol levels ${ }^{(9)}$.

Statins are also known to have antiinflammatory properties and other pleiotropic actions on free oxygen radical formation, smooth muscle cell proliferation, and immunomodulatory effects $^{(\mathbf{1 0})}$.

These pleiotropic properties may prevent the hostile uterine environment associated with preeclampsia and in doing so, prevent the fetal programming of long-term adverse metabolic profile associated with preeclampsia exposure ${ }^{(11)}$.

\section{Aim of Work}

The aim of this work is to study the effect of pravastatin given for primigravidae from $13^{\text {th }}$ week to $16^{\text {th }}$ week of gestation on the incidence of preeclampsia compared to a control group in Egyptian women at EL Sayed Galal Hospitals' obstetric outpatient clinic.

\section{Subjects and methods}

\section{Study design}

This is a prospective case control study of 400 pregnant females presenting for routine antenatal care at $13^{\text {th }}$ to $16^{\text {th }}$ weeks' gestation.

\section{Study place}

A case control study of 400 pregnant females attending the antenatal care clinics at AlHussein University Hospital and EL Sayed Galal Maternity Hospital selected according to the following inclusion and exclusion criteria.

Inclusion criteria: these include the following: Primigravidae, female age: ranges from 20 to 35 years, singleton, viable pregnancy morphologically normal by ultrasound, gestational duration: from $13^{\text {th }}$ weeks to $16^{\text {th }}$ weeks of gestation, average Body mass index (BMI): $20-25 \mathrm{~kg} / \mathrm{m}^{2}$, non-smoker, nonalcoholic, no previous history of hypertension

Exclusion criteria: the following cases were excluded:

- Women with multiple pregnancies.

- Pregnancy duration less than 13 weeks. Or more than 16 weeks of gestation

- Cases with major fetal anomalies

- Women with chronic hypertension evidenced by :

- Blood pressure $\geq 140 / 90 \mathrm{mmHg}$ on two different occasions at least 4 hours apart.

- Those taking anti-hypertensive drugs

- Women with history of recurrent early pregnancy loss.

- Women with proteinuria discovered by urine dipstick test done at first visit.

- Women with contraindication to pravastatin (e.g. hypersensitivity to the active substance or to any of the excipients or active liver disease including unexplained persistent elevations of serum transaminase elevation exceeding $3 \mathrm{x}$ the upper limit of normal)

- Women with known medical conditions:

- Diabetes mellitus

- Sickle cell disease

- Thrombophilias

- Connective tissue diseases

- Anti-phospholipid antibody syndrome

All the patients were submitted to the following steps

1-Informed consent was taken from each patient

2-Full history

3-Blood pressure measurement

4-Albumin in urine test

5-Height and weight measurement for estimation of BMI (Body mass index)

6-General examination

7-Abdominal examination 
8-Obstetric ultrasound for checking the number of fetuses, viability, gestational age, placental location and congenital fetal malformations

The present study includes 400 cases have been randomly divided into two groups (randomization has been done by sealed envelope):

\section{Group A (The study group): (200 case)}

In this group Pravaststin (Lipostat ${ }^{\circledR} 10 \mathrm{mg}$ GSK) was given once daily for 4 weeks starting from the $13^{\text {th }}$ week to the $16^{\text {th }}$ week of gestation.

\section{Group B (The control group): (200 cases)}

In this group placebo was given once daily for 4 weeks starting from the $13^{\text {th }}$ week to the $16^{\text {th }}$ week of gestation

\section{Collected data:}

Patients were asked to complete a questionnaire on maternal age, cigarette smoking during pregnancy (yes or no), method of conception (spontaneous or assisted), medical history (including chronic hypertension, diabetes mellitus, anti-phospholipid syndrome, thrombophilia, and sickle cell disease), medication (including antihypertensive, antidepressant, antiepileptic, aspirin, steroids, betamimetics, insulin and thyroxin), parity (parous or nulliparous if no delivery beyond 24 weeks), obstetric history (including previous pregnancy with preeclampsia), and family history of preeclampsia (mother). The questionnaire was reviewed by a doctor together with the patient. The maternal weight and height were measured, and the BMI was calculated in kilograms per meter squared. The baseline blood pressure was measured and documented and an initial urine dipstick test was done to exclude proteinuria.

\section{Follow up:}

All cases were followed regularly once monthly for the first six months of pregnancy, twice monthly during the seventh and eighth month and then once weekly till delivery.

In follow up visits, the blood pressure was taken by mercurial sphygmomanometers which were calibrated before and at regular intervals during the study. Recordings were taken with the women in the seating position, and 24-h collection of urine was analysed for proteinuria or dipstick analysis of midstream urine specimens was done if 24-h collection of urine was not available.

\section{Outcome measures}

The primary outcome
The definition of preeclampsia was that of the International Society for the Study of Hypertension in Pregnancy. The diastolic blood pressure should be $\geq 90 \mathrm{mmHg}$ on at least two occasions $4 \mathrm{~h}$ apart, developing after 20 weeks of gestation in previously normotensive women in the presence of significant proteinuria of $\geq 300 \mathrm{mg}$ in $24 \mathrm{~h}$ or two readings of at least (+) on dipstick analysis of midstream or catheter urine specimens if no $24-\mathrm{h}$ collection is available. ${ }^{(\mathbf{1})}$

\section{The secondary outcome}

1-Getational age at time of delivery

2-Type of delivery

3-Fetal birth weight

4-Fetal birth defects

5-NICU admission and Neonatal mortality

\section{Statistical Analysis}

Results have been statistically described in terms of mean \pm standard deviation (mean $\pm \mathrm{SD}$ ), frequencies (number of cases) and relative frequencies (percentages). Comparison of quantitative variables between the study groups was done using Student $t$-test for independent samples. Statistical significance was evaluated using the Chi-square test for differences in qualitative variables. A probability value ( $p$ value) less than 0.05 was considered statistically significant, P-value $<0.001$ was considered as highly significant and P-value >0.05 was considered insignificant. All statistical calculations were done using computer programs(Microsoft Corporation, NY, USA) and SPSS (Statistical Package for the Social Science; SPSS Inc., Chicago, IL, USA).

\section{Ethical comments}

This study was ethically approved by the department and full informed consent (oral and written) was obtained from the participants prior to the study.

\section{Results}

In our study, in the group supplemented with pravastatin, 6 of them developed preeclampsia; 4 of them showed severe disease. On the other hand 16 women in the placebo group developed preeclampsia; 11 of them showed severe disease compared to nital the pravastatin group, suggesting a beneficial effect of pravastatin in preventing the onset of preeclampsia.

Birth weight was similar in both groups and 1 case of congeabnormality (Spina bifida) in the study group and 2 cease of congenital abnormalities (Ambigious genitalia and patent ductus arteriosus) in the control group. No maternal, fetal or neonatal death was observed. 
Mohamed Hassanain et al.

Table (1): Comparison between control and cases according to demographic data.

\begin{tabular}{|l|c|c|c||}
\hline Demographic Data & $\begin{array}{c}\text { Control } \\
(\mathbf{N}=\mathbf{2 0 0})\end{array}$ & $\begin{array}{c}\text { Cases } \\
(\mathbf{N}=200)\end{array}$ & p-value \\
\hline \hline Age (years) & $20-35$ & $20-35$ & \\
Range & $28.88 \pm 3.47$ & $27.83 \pm 3.34$ & $0.002^{*}$ \\
Mean \pm SD & & & \\
\hline BMI (wt/(ht)^2) & $20-25$ & $20-25$ & $>0.05$ \\
Range & $23.63 \pm 2.84$ & $23.10 \pm 2.77$ & \\
Mean \pm SD & $60-85$ & $60-85$ & $0.002^{*}$ \\
\hline Body weight & $76.13 \pm 7.94$ & $73.50 \pm 8.50$ & 20 \\
Range & & & \\
Mean \pm SD &
\end{tabular}

t-Independent Sample t-test; p-value >0.05 NS

This table shows no statistically significant difference between groups according to demographic data.

Table (2): Comparison between control and cases according to blood pressure.

\begin{tabular}{|l|c|c|c||}
\hline Blood Pressure & $\begin{array}{c}\text { Control } \\
(\mathbf{N = 2 0 0})\end{array}$ & $\begin{array}{c}\text { Cases } \\
(\mathbf{N}=200)\end{array}$ & p-value \\
\hline \hline Systolic blood pressure & $100-130$ & $100-130$ & \\
Range & $120.75 \pm 7.49$ & $118.13 \pm 8.18$ & $0.001^{*}$ \\
Mean \pm SD & & $60-90$ & \\
Diastolic blood pressure & $60-90$ & $79.80 \pm 8.58$ & $>0.05$ \\
Range & $78.75 \pm 9.4$ & \\
Mean \pm SD &
\end{tabular}

t-Independent Sample t-test; p-value >0.05 NS

This table shows no statistically significant difference between groups according to blood pressure.

Table (3): Comparison between control and cases according to preeclampsia.

\begin{tabular}{||l|c|c|c||}
\hline Preeclampsia & $\begin{array}{c}\text { Control } \\
(\mathbf{N}=\mathbf{2 0 0})\end{array}$ & $\begin{array}{c}\text { Cases } \\
(\mathbf{N}=200)\end{array}$ & p-value \\
\hline \hline Yes & $16(8 \%)$ & $6(3 \%)$ & \multirow{2}{*}{$0.028 *$} \\
\hline
\end{tabular}

$x^{2}$-Chi-square test; $p$-value $<0.05 \mathrm{~S}$

This table shows statistically significant difference between groups according to preeclampsia.

Table (4): Comparison between control and cases according to level of preeclampsia.

\begin{tabular}{|l|c|c|c|}
\hline Level of Preeclampsia & $\begin{array}{c}\text { Control } \\
(\mathbf{N}=\mathbf{1 6})\end{array}$ & $\begin{array}{c}\text { Cases } \\
(\mathbf{N}=6)\end{array}$ & \multirow{2}{*}{ p-value } \\
\hline \hline Mild & $5(31.2 \%)$ & $2(33.3 \%)$ & \multirow{2}{*}{$>0.05$} \\
\hline Severe & $11(68.8 \%)$ & $4(66.7 \%)$ & \\
\hline
\end{tabular}

$x^{2}$-Chi-square test; p-value $>0.05 \mathrm{NS}$

This table shows no statistically significant difference between groups according to level of preeclampsia.

Table (5): Comparison between control and cases according to 2ry (Fetal) outcomes

\begin{tabular}{|l|c|c|c|}
\hline 2ry (Fetal) outcome & $\begin{array}{l}\text { Control } \\
(\mathbf{N}=200)\end{array}$ & $\begin{array}{c}\text { Cases } \\
(\mathbf{N}=200)\end{array}$ & p-value \\
\hline
\end{tabular}


Effect of Pravastatin on the Incidence of Preeclampsia

\begin{tabular}{|l|c|c|c|}
\hline Congenital fetal malformations & $1(6.25 \%)$ & $2(1.0 \%)$ & $>0.05$ \\
\hline Gestational age at time of delivery & $34 \_40$ weeks & $35 \_40$ weeks & \\
\hline Fetal weight at time of delivery & $2.5-3.5 \mathrm{~kg}$ & $2.5-3.5 \mathrm{~kg}$ & \\
\hline NICU Admission & 0 & 0 & \\
\hline
\end{tabular}

$x^{2}$-Chi-square test; p-value $>0.05 \mathrm{NS}$

This table shows no statistically significant difference between groups according to 2ry (Fetal) outcomes

Table (6): Comparison between control and cases according to side effect.

\begin{tabular}{|c|c|c|c|}
\hline Side Effect & $\begin{array}{l}\text { Control } \\
(\mathrm{N}=200)\end{array}$ & $\begin{array}{c}\begin{array}{c}\text { Cases } \\
(\mathrm{N}=200)\end{array} \\
\end{array}$ & p-value \\
\hline Heartburn & $2(1.0 \%)$ & $2(1.0 \%)$ & $>0.05$ \\
\hline Musculoskeletal pain & $4(2.0 \%)$ & $3(1.5 \%)$ & $>0.05$ \\
\hline Dizziness & $2(1.0 \%)$ & $2(1.0 \%)$ & $>0.05$ \\
\hline Diarrhea & $1(0.5 \%)$ & $5(2.5 \%)$ & $>0.05$ \\
\hline Headache & $3(1.5 \%)$ & $1(0.5 \%)$ & $>0.05$ \\
\hline Cough & $4(2.0 \%)$ & $2(1.0 \%)$ & $>0.05$ \\
\hline Nausea & $2(1.0 \%)$ & $3(1.5 \%)$ & $>0.05$ \\
\hline
\end{tabular}

$x^{2}$-Chi-square test; $p$-value $>0.05 \mathrm{NS}$

This table shows no statistically significant difference between groups according to side effect.

\section{Discussion}

Hypertensive disorders are among the most common medical complications of pregnancy; the reported incidence is between $5 \%$ and $10 \%{ }^{(1)}$.

Preeclampsia is a pregnancy-specific hypertensive disease with multisystem involvement. It usually occurs after 20 weeks of gestation, most often near term, and can be superimposed on another hypertensive disorder. Preeclampsia, the most common form of high blood pressure (BP) that complicates pregnancy, is primarily defined by the occurrence of new-onset hypertension plus new-onset proteinuria. However, although these two criteria are considered the classic definition of preeclampsia, some women present with hypertension and multi systemic signs usually indicative of disease severity in the absence of proteinuria ${ }^{(\mathbf{1 2})}$.

In the absence of proteinuria, preeclampsia is diagnosed as hypertension in association with thrombocytopenia (platelet count less than 100,000/microliter), impaired liver function (elevated blood levels of liver transaminases to twice the normal concentration), the new development of renal insufficiency (elevated serum creatinine greater than $1.1 \mathrm{mg} / \mathrm{dl}$ or a doubling of serum creatinine in the absence of other renal disease), pulmonary edema, or new-onset cerebral or visual disturbances ${ }^{\mathbf{( 1 2 )}}$.

Hypertensive disorders in pregnancy remain one of the leading cause of maternal death worldwide (about $20 \%$ to $25 \%$ of maternal deaths in developing as well as developed nations) ${ }^{(\mathbf{1 3})}$.

Preeclampsia shares pathogenic similarities with adult cardiovascular diseases as well as many risk factors. Endothelial dysfunction and inflammation are fundamental for the initiation and progression of both. There is strong evidence that 3-hydroxy-3-methylglutaryl-coenzyme A (HMG-CoA) reductase inhibitors (statins) are beneficial in primary and secondary prevention of cardiovascular mortality and other cardiovascular events. Biological plausibility as well as animal data supports a similar role for statins in preeclampsia. Currently, there are no clinically available agents to prevent preeclampsia. However because of the below properties of statins, this class of medications could substantially contribute to preeclampsia prevention.

1. Statins pleiotropic actions on various mechanisms: reversing the angiogenic imbalance by upregulating vascular endothelial growth factor (VEGF) and placental growth factor (PIGF), and reducing the antiangiogenic factors such as soluble fms-like tyrosine kinase-1 (sFlt-1) and soluble endoglin (sEng).

2. Statins up regulation of endothelial nitric oxide synthase, leading to improved nitric oxide production in the vasculature and to activate the heme oxygenase-1/carbon monoxide (HO-1/CO) pathway, protecting the endothelium and reducing the inflammatory and oxidative insults ${ }^{(\mathbf{1 0})}$. 


\section{Mohamed Hassanain et al.}

In humans, the first report to suggest the beneficial effects of pravastatin in preventing preeclampsia described a patient with antiphospholipid syndrome (APS). The APSpatient, with a history of early preeclampsia leading to a still birth at week 26, developed preeclampsia in her second pregnancy, despite anticoagulant treatment. Uterine artery Dopplers showed increased resistance and bilateral notching. To prevent intrauterine fetal death as in the previous pregnancy, the patient was supplemented with pravastatin. Addition of pravastatin (20 $\mathrm{mg} /$ day) to standard of care therapy low molecular weight heparin (LMWH) plus low dose aspirin (LDA) normalised maternal disease, blood pressure and proteinuria and reversed abnormal uterine blood flow. A live and healthy baby girl weighing $2830 \mathrm{~g}$ was delivered vaginally at 38 weeks with no complications. The patient stopped taking pravastatin prior to delivery, in preparation for breastfeeding. Interestingly, preeclampsia relapsed shortly after delivery. Pravastatin therapy ( $20 \mathrm{mg} /$ day) was started again and the preeclamptic features disappeared ${ }^{\left({ }^{(14)}\right.}$.

A pilot clinical study to examine the effects of pravastatin in women diagnosed with $\mathrm{PE}$ between 24 and 29 weeks was conducted in Australia $^{(15)}$.

In this study, 4 patients significantly hypertensive (90-105/155-200 mm Hg) and with proteinuria ranging from 840 to $2990 \mathrm{mg} / 24 \mathrm{~h}$ were treated with daily pravastatin $(40 \mathrm{mg})$ from the day of admission. All women presented growth restricted fetuses. The patients also received betamethasone and magnesium sulfate for lung maturation and neuroprotection. After pravastatin treatment symptoms of PE resolved. Pravastatin stabilized blood pressure in 3 of the patients and only one patient required antihypertensive medication nifedipine. Delivery in these women was triggered by fetal indications rather than worsening of maternal disease. Furthermore, serum sFLT-1, sENG and endothelin-1 levels remained stable after pravastatin treatment. The authors concluded that pravastatin might be a candidate therapeutic strategy for preeclampsia.

Many women with OAPS do not respond LMWH+ LDA and develop PE. In an attempt to improve obstetrical outcome, the effects of pravastatin in this group of women with OAPS was assessed. 21 pregnant women with OAPS and an adverse obstetric history that developed PE and/or severe intrauterine growth restriction (IUGR) despite being treated with LDA + LMWH since the beginning of pregnancy participated in this study.
A group of 10 women with APS and severe PE and/or IUGR received only conventional LDA + LMWH treatment and served as control. Pravastatin ( $20 \mathrm{mg} /$ day) was added to conventional treatment in 11 women when signs of preeclampsia and or IUGR were observed ${ }^{(\mathbf{1 4})}$.

In the group supplemented with pravastatin, placental blood flow increased and hypertension and proteinuria stabilized as early as 10 days after treatment leading to live birth in all patients. In the control group that received only antithrombotic therapy, maternal disease did not improve and deliveries occurred preterm and only 6 of the 11 neonates survived. Neonates spent several months at the neonatology intensive (NICU) care unit and 3 show abnormal development. In the group treated with pravastatin pregnancies were significantly prolonged after diagnosis (13 weeks compared to the group that only received LMWH+ LDA 4.5 weeks. Delivery dates in the group supplemented with pravastatin were close to term allowing appropriate fetal development and preventing admission to the NICU. No late sequelae were reported for the infants in the pravastatin group ${ }^{(\mathbf{1 4})}$.

This study suggests that women with refractory OAPS may have improved pregnancy outcomes with pravastatin taken at the time of onset of PE or severe IUGR until the end of pregnancy. The studies mentioned above were aimed to ameliorate preeclampsia after the onset of the disease. Interestingly, a study by Costantine et al., investigated the effectiveness of pravastatin in preventing preeclampsia in high risk patients. This initial pilot study in a multicenter, double-blind, placebo-controlled, randomized trial, evaluated the utility of pravastatin in preventing preeclampsia in women with a history of severe preeclampsia in a prior pregnancy that required delivery before 34 weeks. Twenty subjects between 12 and 16 weeks of pregnancy, were randomized to pravastatin (10 $\mathrm{mg})(\mathrm{n}=10)$ or placebo $(\mathrm{n}=10)$. Four women in the placebo group developed preeclampsia; three of them showed severe disease compared to the pravastatin group, suggesting a beneficial effect of pravastatin in preventing the onset of preeclampsia (10).

In line with the proangiogenic effects of pravastatin, a slight but not significant diminution in anti angiogenic factors sFlt-1 and sEng and increase in PlGF was observed in the pravastatin group compared to placebo. Birth weight was similar in both groups and no congenital abnormalities or developmental abnormalities 
were observed in the two groups. No maternal, fetal or neonatal death was observed ${ }^{(\mathbf{1 0})}$.

As previously stated, this study provided important information regarding preliminary safety and pharmacokinetic data regarding the use of pravastatin in early pregnancy to prevent preeclampsia in high risk women. This pilot study was part of a larger randomized controlled study (RCT) that is currently taking place at Eunice Kennedy Shriver National Institute of Child Health and Human Development Obstetric-Fetal Pharmacology Research Units Network. A proof of principle, double-blind, randomised placebocontrolled, multicentre trial of pravastatin to ameliorate early onset pre-eclampsia (sTAmP) was recently completed in the United Kingdom. The aim of this trial was to establish whether a significant reduction of angiogenic markers by pravastatin will alleviate the severity of early-onset pre-eclampsia in women ${ }^{(\mathbf{1 6})}$.

The preclinical studies showed promising results on the beneficial effects of pravastatin in treating placental insufficiency and laid foundation for the human studies. The mouse studies and the pilot human studies emphasize the need of a randomised clinical trials (RCT) to confirm these observations. Pravastatin was never included in category $\mathrm{X}$ by the FDA and due to its hydrophilic characteristics has a minimal transplacental transfer, diminishing fetal safety concerns. The new FDA classification that replaced the five-letter system together with the epidemiological studies demonstrating that pravastatin exposure during pregnancy does not affect fetal development indicate that the use of pravastatin is safe during pregnancy. Other therapeutic strategies to prevent and/or treat preeclampsia have had limited success and the only current available therapy is the delivery of the baby, associated with high risks due to prematurity. Thus, pravastatin is a promising option to prevent and treat preeclampsia. RCT should confirm the effectiveness of pravastatin ${ }^{(16)}$.

\section{Conclusion:}

Pravastatin can be used as an effective agent in the prevention of preeclampsia in newborns within neonatal period including fetal weight, gestational age at time of delivery and NICU admission. However, extended follow up is needed till the age of 5 years to assess cognitive functions and school performance among children born for women who received pravastatin for prevention of preeclampsia.

\section{Recommendations:}

More research is needed to evaluate newer generations of statins (simvastatin and rouvastatin) regarding prevention of preeclampsia and safety on fetal outcome.

\section{References}

1- American College of Obstetricians and Gynecologists (2013) : Task Force on Hypertension in Pregnancy. Hypertension in pregnancy. Report of the American College of Obstetricians and Gynecologists' task force on hypertension in pregnancy. Obstet Gynecol., 122:11221131.

2- Yu., G. C.S. Smith, A. T. Papageorghiou, A. M. Cacho, and K. H. Nicolaides (2008) : "An integrated model for the prediction of preeclampsia using maternal factors and uterine artery Doppler velocimetry in unselected low-risk women, "American Journal of Obstetrics and Gynecology, 193(2): 429-436.

3- Poon LC, Nicolaides KH (2014) : Firsttrimester maternal factors and biomarker screening for preeclampsia. Prenat Diagn., 34:618.

4- McLean, Buckett L, Davidson R, I .(2015) : Preclinical and clinical pharmacology of Pravastatin, a new 3hydroxy-3- methylglutaryl coenzyme A reductase inhibitor. Am $\mathrm{J}$ Cardiol. 87(5A):28B-32B.

5- Mc Taggart F, Buckett $L$, Davidson $R$ (2001) : Preclinical and clinical pharmacology of Rosuvastatin, a new 3hydroxy-3- methylglutaryl coenzyme A reductase inhibitor. Am J Cardiol., 87(5A):28B-32B

6- Edison RJ, Muenke M (2004) : Mechanistic and epidemiologic considerations in the evaluation of adverse birth outcomes following gestational exposure to statins. Am J Med Genet A., 131 (3):287-298.

7- Petersen EE, Mitchell AA, Carey JC, Werler MM, Louik C, Rasmussen SA (2008): Maternal exposure to statins and risk for birth defects: a case-series 
Mohamed Hassanain et al.

approach. Am J Med Genet A. 146A(20):2701-2705.

8- Taguchi N, Rubin ET, Hosokawa A (2008) : Prenatal exposure to HMG-CoA reductase inhibitors: effects on fetal and neonatal outcomes. Reprod Toxicol. ,26(2):175-177

9- Saad ,Tamayo E, Lu F, Bytautiene E, Longo M, Hankins GD(2014) : Diagnosis, prevention, and management of eclampsia.Obstetrics \& Gynecology, 105: 402-410, 2014.

10- Costantine M, Tamayo E, Bytautiene E (2013) : Using pravastatin to improve the vascular reactivity in a mouse model of soluble Fms-like tyrosine kinase-1-induced preeclampsia. 116(116):114-120.
11- Elahi, Fausett MB, Fraser A (2008) : Paternal and maternal components of the predisposition to preeclampsia. $\mathrm{N}$ Engl $\mathbf{J}$ Med.,344:867.

12- Gifford R, August P, Cunningham G (2002) : Report of the national high blood pressure education program working group on high blood pressure in pregnancy. American Journal of Obstetrics and Gynecology, 183: S1-S22, 2002.

13- Brown , Mackenzie C, Dunsmuir W (2006) : Can we predict recurrence of preeclampsia or gestational hypertension? BJOG., 114:984.

14- Lefkou E, Mamopoulos A, Fragakis N, Dagklis T, Vosnakis C, Nounopoulos E, Rousso D, Girardi G(2014) : Clinical improvement and successful pregnancy in a preeclamptic patient with antiphospholipid syndrome treated with pravastatin. Hypertension, 63:e118-e119.

15- Brownfoot FC, Hannan N, Onda K, Tong S, Kaitu'u-Lino T(2014) : Soluble endoglin production is upregulated by oxysterols but not quenched by pravastatin in primary placental and endothelial cells. Placenta,35:724-731.

16- Marzoa-Rivas R, Crespo-Leiro MG, Paniagua-Marin MJ(2005) : Safety of statins when response is carefully monitored: a study of 336heartrecipients. Transplant Proc., 37(9):4071-4073. 